Results: There were 41 SLE patients integrated in the study, female:male ratio $9,25: 1$, mean age (SD) 39 (12.35) years (range 20-67 years), disease duration (SD) was 9.92 (9.18) month (range 1-24). The mean disease activity by SLEDAI was 11.2 \pm 7.84 (range 2-34) and SLAM - 8.83 \pm 4.41 (range 3-22) points, both indices denoted high disease activity level. Mean PGA values were 48.93 (19.13) (range 10-80), and mean MDGA values 45 (19.04) (range 10-80). Also, PGA and MDGA didn't correlate with SLEDAI $(r=0,25, p>0,05 ; r=0,27, p>0,05)$, while a statistically significant correlation was determined with SLAM index $(r=0,85$, $p<0.001 ; r=0,46, p=0.002$ ). A subclass analysis of SLAM components showed that cortical dysfunction (depression, psychosis) and the presence of headache correlated with PGA $(r=0,36, p<0,05 ; r=0,4, p<0,05)$, so we can establish that the difference in correlation between SLAM and SLEDAI with PGA and MDGA is explained by a more accurate disease assessment by SLAM, including also subjective complaints that influences the global patient's status.

Conclusions: The use of SLAM for disease activity assessment in early SLE patients is more sensible than SLEDAl and its results correlates with PGA and MDGA.

Disclosure of Interest: None declared

DOI: 10.1136/annrheumdis-2017-eular.3710

\section{SAT0297 SLE PATIENTS WITH SECONDARY SJÖGREN'S SYNDROME ARE CHARACTERIZED BY TYPICAL AUTOANTIBODIES AND A PRO-INFLAMMATORY STATE}

M. Kvarnström ${ }^{1}$, G. Ruacho ${ }^{2}$, J.T. Gustafsson ${ }^{3}$, A. Zickert ${ }^{3}$, V. Oke ${ }^{3}$, J. Rönnelid ${ }^{4}$, K. Elvin ${ }^{5}$, I. Gunnarsson ${ }^{3}$, E. Svenungsson ${ }^{3} .{ }^{1}$ Dep. of Medicine, Unit of Experimental Rheumatology, Karolinska Institutet, Karolinska Uniververity Hospital, Stockholm; ${ }^{2}$ Centre for Clinical Research Sörmland, Uppsala Univerity, Sörmland; ${ }^{3}$ Dep. of Medicine, Unit of Rheumatology, Karolinska Institutet, Karolinska Uniververity Hospital, Stockholm; ${ }^{4}$ Department of Immunology, Genetics and Pathology, Uppsala Univerity, Uppsala; ${ }^{5}$ Unit of Clinical Immunology, Department of Clinical Immunology and Transfusion Medicine, Karolinska Institutet, Karolinska Uniververity Hospital, Stockholm, Sweden

Background: Sjögren's syndrome occurs in isolation (primary Sjögren's syndrome, pSS), but it is also often secondary (sSS) to, and sometimes difficult to delineate from, other rheumatic diseases, in particular from systemic lupus erythematosus (SLE). Consequently there is a need to investigate similarities and differences between SLE patients with (SLE-sSS) and without SSS (SLE-noSS). Objectives: To investigate the occurrence of SSS in a large cohort of SLE patients and to explore clinical and laboratory characteristics associated with SLE-sSS as compared to SLE-noSS and controls.

Methods: We included 504 consecutive SLE patients and 322 population controls, individually matched for age and gender to the first patients. All patients fulfilled the 1982 revised ACR criteria for SLE. SLE-sSS was defined according to the American-European consensus criteria (AECC). Accordingly, subjective and

Immunoglobulins, autoantibodies and pro-inflammatory cytokines in SLE-SS, SLE-noSS and population controls

\begin{tabular}{|c|c|c|c|c|}
\hline & $\begin{array}{c}\text { Controls } \\
\mathrm{N}=322 \\
\text { median (lQR) or } \\
N(\%)\end{array}$ & $\begin{array}{c}\text { SLE-SS } \\
N=117 \\
\text { medtian (1QR) or } \\
N(\%)\end{array}$ & $\begin{array}{c}\text { SLE-noSS } \\
\text { N=387 } \\
\text { median (1QR) ar } \\
\text { N( } \% \text { ) }\end{array}$ & $\begin{array}{l}\text { p-value } \\
\text { St.E-SS } \\
\text { ns. } \\
\text { SLE-noss }\end{array}$ \\
\hline IgA total $\mathrm{g} / \mathrm{L}$ & $2.1(1.5 .2 .8)$ & $29(1.8-4.3)$ & $2,7(1.9 .3 .6)$ & 0.38 \\
\hline IgG total g/L & $10.9(9.5 \cdot 12.2)$ & $14.5(10.4-18.3)$ & $12.4(9.8 \cdot 15.8)$ & 0009 \\
\hline IgM total g/L & $1.1(08.1 .6)$ & $1.0(0.5 \cdot 1.6)$ & $0.9(0.6 .15)$ & 2.59 \\
\hline $\begin{array}{l}\text { anti-dsDNA \% positive } \\
(+)\end{array}$ & $5(1.6)$ & $36(313)$ & $154(41)$ & 0.06 \\
\hline anti-R052\%+ & $3(0.9)$ & $36(47.9)$ & $84(21.8)$ & 00001 \\
\hline anti-Ro60\% + & $5(1.6)$ & $69(59)$ & $137(35.9)$ & $\infty .0001$ \\
\hline anti-La/SSB $\%+$ & $10(3.1)$ & $44(376)$ & ब्रा8) & $\infty 0001$ \\
\hline anti-Sm \% + & $1(0.3)$ & $19(16.2)$ & $75(19.5)$ & 0.42 \\
\hline anti-RNP $68 \%+$ & $0(0)$ & $119.4)$ & $40(10,4)$ & 0.74 \\
\hline RrIgG $\%+$ & $10261(3.8)$ & $17 / 80(212)$ & $35 / 25 \times(13.5)$ & 200 \\
\hline RfIgM \%+ & $14283(4.9)$ & $3283(386)$ & $56 / 281(19.9)$ & 0.0005 \\
\hline RfIgA \%+ & $12 / 282(12.4)$ & $34774(45.9)$ & $73 / 267(28.0)$ & 0.004 \\
\hline TNF- $\alpha \mathrm{pg} / \mathrm{mL}$ & $2.3(20.2 .8)$ & $4.9(3.6-7.1)$ & $4.4(3.0 .6 .0)$ & 0.000 \\
\hline IL-6 $\mathrm{pg} / \mathrm{mL}$ & $0.5(0.4 .0 .7)$ & $1.5(0.8 .3 .0)$ & $1.1(0.6 \cdot 2.0)$ & 0.009 \\
\hline MCP.4 pg/mL & $55.8(40.8 .80 .5)$ & $94.9(66.9 .131 .3)$ & $74.7(52.4 .1200)$ & 0.019 \\
\hline MIP-1 B pg/mL & $43.7(3 \times 4.564)$ & $81.1((54.8-123.6)$ & $68.9(50.3 \cdot 105.1)$ & 0.021 \\
\hline $1 \mathrm{IL} .12 / 1 \mathrm{~L}-23 \mathrm{p} 40 \mathrm{pg} / \mathrm{mL}$ & $131.2(99.8 .1795)$ & $2113(141.4-363,8)$ & $177.1(119.62745)$ & 0.032 \\
\hline $1 \mathrm{P}-10 \mathrm{pg} / \mathrm{mL}$ & $351.9(259.2-476.4)$ & $805(536-1911)$ & $726(+40.1471)$ & 0.036 \\
\hline
\end{tabular}

objective quantifications of sicca symptoms were recorded on all subjects. All underwent a thorough clinical investigation. SLE-associated autoantibodies, (ANA screening by BioPlex 2200 system, Bio-Rad) and Rheumatoid factor (RF, Phadia Immunocap 250) were determined with standardized methods for all subjects, Routine laboratory workup and a panel of cytokines (MSD 30-plex cytokine assays, performed on samples from 433 consecutive SLE patients and 319 controls) were measured on fasting blood samples.

Results: SLE-SSS, as defined by AECC, occurred in $23 \%$ of the SLE patients. Compared to SLE-noSS the SLE-sSS group was older, both at inclusion (55 vs $43 y r s, p<0.0001)$ and at disease onset ( 40 vs. 32 yrs $p<0.0001)$, and with a greater number of females (96 vs. $83 \%, p=0.0007$ ), higher occurrence of leucopenia ( 57 vs. $45 \%, p=0.02$ ) and peripheral neuropathy ( 15 vs $7 \%, p=0.01$ ). Nephritis was less common in SLE-sSS (32 vs $43 \%, p=0.03$ ). Higher levels of total IgG, positivity for anti-SSA/Ro52, anti-SSA/Ro60, anti-SSB antibodies, RF IgM and RF IgA further characterized the SLE-sSS group. 20/30 investigated cytokines were detectable, of these 19/20 were higher in SLE than in controls. 6/20 cytokines (TNF-a, IL-6, MCP-4, MIP-1 $\beta$, IL12/IL-23p40 and IP-10) were upregulated in SLE-sSS vs. SLE-noSS (see table for figures).

Conclusions: Through strictly applying the AECC criteria we report that the frequency of SLE-SSS increases with age and affects roughly $1 / 4$ of SLE patients. Nephritis was less common while leucopenia and peripheral neuropathy were more common among SLE-sSS patients. In addition to excess of well-known SS-associated autoantibodies we report higher levels of six pro-inflammatory cytokines in SLE-sSS as compared to SLE-noSS. These findings demonstrate that, though often regarded as a milder version of SLE, patients with SLE-SSS are characterized by a state of chronic systemic inflammation.

Acknowledgements: Susanna Eketjäll at Cardiovascular and Metabolic Diseases, Innovative Medicines and Early Development Biotech Unit, AstraZeneca, Integrated Cardio Metabolic Centre (ICMC), Karolinska Institutet, Huddinge, Sweden

Disclosure of Interest: None declared

DOI: 10.1136/annrheumdis-2017-eular.5449

\section{SAT0298 INFLUENCE OF AGE ONSET IN CLINICAL AND BIOLOGICAL SPECTRUM OF SYSTEMIC LUPUS ERYTHEMATOSUS}

M. Kechida ${ }^{1}$, N. Lorenzo ${ }^{2}$, I. Ben Chaaben ${ }^{1}$, R. Klii ${ }^{1}$, S. Hammami ${ }^{1}$ I. Khochtali ${ }^{1}{ }^{1}$ Internal Medicine and Endocrinology Department, Fattouma Bourguiba Hospital, Monastir, Tunisia; ${ }^{2}$ Internal Medicine and cancerology Department, Saint-Cyr Hospital, Lyon, France

Background: Systemic lupus erythematosus (SLE) is a multi systemic auto immune disease which can affect patients at any age.

Objectives: We aimed to study influence of age onset in clinical and biological spectrum of SLE

Methods: medical records of 89 patients diagnosed as SLE according to the ACR criteria of 1997, between January 2004 and December 2016, were retrospectively analyzed. Patients were divided into 3 groups according to the age of onset: Juvenile onset patients (group 1) (G1) ( $\leq 16$ years), Adult onset patients (group 2) (G2) ( $>16$ and $<50$ years), Late onset patients (group 3) (G3) ( $\geq 50$ years). Clinical and biological comparative study was conducted between the 3 groups. Data were analyzed by chi-square test and potentially associated factors were tested by binary logistic regression.

Results: among the patients $11.2 \%$ are in G1, $75.3 \%$ in $\mathrm{G} 2$ and $13.5 \%$ in G3. Prevalence of SLE was higher in female than male $(F / M=9 / 1)$ but predominance of women was lower in $\mathrm{G} 1$ ( $\mathrm{F} / \mathrm{M}=4 / 1)$ compared to $\mathrm{G} 2(\mathrm{~F} / \mathrm{M}=10 / 1)$ and $\mathrm{G} 3$ $(\mathrm{F} / \mathrm{M}=11 / 1)$. Patients in $\mathrm{G} 3 \mathrm{had}$ more hypertension $(41.7 \%)$ compared to $\mathrm{G} 2(6 \%)$ $(\mathrm{p}=0.5)$ and $\mathrm{G} 1(0 \%)(\mathrm{p}=0.04)$. Vespertilio erythema was less frequently found in G3 $(33.3 \%)$ compared to $\mathrm{G} 2(64.2 \%)(\mathrm{p}=0.045)$ and $\mathrm{G} 1(80 \%)(\mathrm{p}=0.04)$. Anti Sm antibodies were more frequent in $\mathrm{G} 1(87.5 \%)$ compared to $\mathrm{G} 2(38.5 \%)(\mathrm{p}=0.009)$ and G3 (18.2\%) ( $\mathrm{p}=0.003)$. Multivariate analysis showed that hypertension is significantly associated to late onset lupus $(\mathrm{OR}=29,95 \% \mathrm{IC}=[2.77-320], \mathrm{p}=0.05)$ and anti $\mathrm{Sm}$ antibody is more frequent in juvenile onset patients (OR=12, 95\% $\mathrm{IC}=[1.4-117], \mathrm{p}=0.024)$.

Conclusions: according to our study, prevalence of lupus is higher in female regardless of age onset. Late onset lupus is associated to a high frequency of co morbidity while anti Sm antibody seems to be a hallmark of juvenile onset.

Disclosure of Interest: None declared

DOI: 10.1136/annrheumdis-2017-eular.3003

\section{SAT0299 ATYPICAL ANTIBODIES IN PATIENTS WITH PRIMARY SJOGREN'S SYNDROME}

M. Fernandez Castro ${ }^{1}$, J.L. Andreu ${ }^{2}$, C. Sanchez-Piedra ${ }^{3}$, V. Martínez Taboada $^{4}$, A. Olive ${ }^{5}$, J. Rosas ${ }^{6}$ on behalf of SJOGRENSER group, part of the Spanish Society of Rheumatology Systemic Autoimmune Diseases Study Group (EASSER). ${ }^{1}$ Rheumatology, Hospital Infanta Sofía; ${ }^{2}$ Rheumatology, Hospital Puerta de Hierro Majadahonda; ${ }^{3}$ Research unit, Spanish Society of Rheumatology, Madrid; ${ }^{4}$ Rheumatology, Hospital Marque's de Valdecilla, Santander; ${ }^{5}$ Rheumatology, Hospital Hospital Germans Trias i Pujol, Barcelona; ${ }^{6}$ Rheumatology, Hospital Hospital Marina Baixa, Alicante, Spain

Background: One of the main features of primary Sjögren's syndrome (pSS) is 